\title{
Erythema nodosum - review of the literature
}

\author{
Matgorzata Chowaniec, Aleksandra Starba, Piotr Wiland
}

Department of Rheumatology and Internal Diseases, University Hospital in Wroclaw, Wroclaw, Poland

\begin{abstract}
Erythema nodosum is the most common form of panniculitis. It may have many aetiological factors. Erythema nodosum occurs three to five times more often in female patients. It appears as erythematous painful rounded nodules, located most often on the anterior surface of the lower extremities, and may be accompanied by systemic symptoms such as fever, malaise and arthralgia. During diagnosis, oncological vigilance should be maintained, because erythema nodosum may be a paraneoplastic symptom. It requires an interdisciplinary approach and exclusion of many underlying causes.
\end{abstract}

Key words: erythema nodosum, panniculitis, clinical image.

\section{Introduction}

Erythema nodosum (EN) is the most common clinical form of panniculitis (inflammation of subcutaneous fat tissue). It appears as erythematous painful rounded lumps, usually 1-6 cm in diameter. Erythema nodosum is almost always located symmetrically on the anterior surface of the lower extremities, but can also spread to the thighs, arms and neck. These lesions do not have a tendency to necrosis and resolve spontaneously within 2-8 weeks without leaving scars. It may be accompanied by systemic symptoms such as fever, malaise and arthralgia [1-4].

The correlation with the location of erythema nodosum on the shins is unknown. It is presumed that it is related to local anatomical circumstances. The skin of the extensor surface of the lower legs is characterized by a lack of underlying muscle pump and unique coexistence of relatively poor arterial blood supply, rich lymphatic system and difficult venous return caused by gravitational effects. This combination can cause difficulties in elimination of the source of inflammation [5].

Erythema nodosum is more common in women and occurs three to five times more often in female patients [4]. It can occur in all age groups, but it is typically seen between the second and the fourth decades of life. The higher prevalence of erythema nodosum among young people is considered to be due to the higher incidence of sarcoidosis in this age group. Moreover, it is considered that the increased incidence of erythema nodosum in the first half of the calendar year is associated with more frequent streptococcal infection aetiology for this time of year. In addition, family history of erythema nodosum is usually associated with infectious aetiology [5].

The histopathological signs of erythema nodosum are characterized by septal inflammation of subcutaneous fat tissue (septal panniculitis). Haemorrhages can be seen in the early phase of erythema nodosum as a result of infiltration of neutrophils around proliferating capillaries. What is important, erythema nodosum may appear without signs of vasculitis, but inflammation as well as haemorrhages may occur within the small vessels. Characteristic histopathological findings are so-called Miescher's radial granulomas. They consist of nodular aggregations of small histiocytes radially surrounding blood vessels [1].

\section{Review of the literature}

Erythema nodosum may have a different aetiological factors. It depends on the population and the geographical latitude. The most common causes are infections (28-48\%), sarcoidosis (11-25\%), drugs (3-10\%), pregnancy (2-5\%) and enteropathies (1-4\%). However,

Address for correspondence:

Małgorzata Chowaniec, Department of Rheumatology and Internal Diseases, University Hospital in Wroclaw, Borowska 213,

50-556 Wroclaw, Poland, e-mail: margaret.t_m@op.pl

Submitted: 13.03.2016; Accepted: 22.04.2016 
in many cases it is impossible to determine the cause (idiopathic erythema nodosum) $[1,6]$.

There are reports that erythema nodosum, particularly the recurrent form with elevated markers of inflammation, may be the only manifestation of vasculitis. For example, the frequency of skin lesions characteristic for erythema nodosum in the case of Behçet diseases is about 50\%, while in Takayasu arteritis it is assessed as 6-19\% [1, 7-9]. Lesions similar to erythema nodosum, besides ulceration of the oral mucosa, may occur before the organ manifestation of Behçet disease. It is believed that vasculitis dominates the histopathological image of erythema nodosum in the case of Behçet disease, in contrast to the classic image of erythema nodosum [10, 11]. Otherwise during diagnosis, oncological vigilance should be maintained, because erythema nodosum may be a paraneoplastic symptom appearing mostly in the course of lymphoma and leukaemia [12].

\section{Infectious agent}

Streptococcal throat infection is the most common cause of EN at our latitude [6]. Erythema nodosum appears generally 2-3 weeks after resolution of pharyngitis. An elevated antistreptolysin $\mathrm{O}$ level is often observed. However, while skin lesion occurs, the pharyngeal swab is negative. Other infectious aetiological agents are Yersinia spp., Mycoplasma, Chlamydia, Histoplasma, Coccidioides and Mycobacterium. The prevalence of tuberculosis in advanced countries such as the US is low, and streptococcal infections and sarcoidosis are the main causative factors, but in regions such as Thailand, India, Turkey or South Africa, Mycobacterium tuberculosis may be the most important factor in EN $[13,14]$.

\section{Sarcoidosis}

Sarcoidosis is the second most common cause of EN. Skin eruptions in the course of sarcoidosis are observed in $25 \%$ of patients. Often the lesions are symmetrical on the extensor surface of both lower limbs. Other skin lesions, predominantly chronic, are lupus pernio, maculopapular lesions, sarcoid discs and scars [15].

Coincidence of such a triad of symptoms as erythema nodosum, arthritis and hilar lymphadenopathy in the course of sarcoidosis is called Löfgren syndrome. This syndrome is usually the early stage of sarcoidosis, which has an acute course and a good prognosis. It is important to remember that the enlargement of the hilar lymph nodes is not specific for Löfgren syndrome and can also occur in such diseases as lymphoma, tuberculosis, coccidioidomycosis, histoplasmosis or acute infections caused by Chlamydia pneumoniae [5].
As proven recently, occurrence of EN in sarcoidosis is related to polymorphism in the promoter region of the TNF gene in the $308^{\text {th }}$ position of both sexes. Additionally, women demonstrated an association with polymorphism of intron 1 in the tumor necrosis factor $\beta$ (TNF- $\beta$ ) gene (lymphotoxin $\alpha$ ) - located adjacent to the TNF- $\alpha$ gene. It is also worth mentioning that oestradiol takes part in up-regulation of TNF, which can potentially be an important factor determining the frequency of developing of EN between the genders. Confirmation of the hypothesis may be the fact that EN occurs with the same frequency in men and women in the prepubertal period. Considering the role of TNF- $\alpha$, it is possible that monoclonal antibody directed against this cytokine may be effective in sarcoidosis treatment [16].

It is considered that biopsy confirmation in patients presenting with classical Löfgren syndrome with symmetrical bilateral hilar adenopathy is usually not necessary. On the other hand, if there is asymmetrical hilar adenopathy or clinical suspicion of either malignancy or tuberculosis, a histopathological examination should be undertaken [17]. To distinguish skin lesions taking tuberculosis into consideration, the QuantiFERON-TB Gold assay can be used, as it is characterized by high sensitivity and specificity, and it detects the MTB antigen-specific interferon $\gamma($ IFN- $\gamma)[14]$.

The use of systemic steroids is a matter of debate and should be considered if underlying conditions such as infection or malignancy have been excluded. Oral prednisone at a dosage of $60 \mathrm{mg}$ every morning is a typical dose (a general rule is $1 \mathrm{mg}$ per kg per day). Patients should be treated until complete resolution of skin lesions [1].

Furthermore, our clinical experience has shown that if the chest radiography is within normal limits but there is a clinical suspicion of sarcoidosis, high-resolution chest computed tomography (HRCT) should be performed. HRCT provides better diagnostic performance of lung alterations such as hilar adenopathy or nodular infiltrates compared to chest X-ray.

\section{Pregnancy and oral contraceptive pills}

The high incidence of EN in females suggests that it is related to sex hormones, confirmed by the more frequent occurrence during pregnancy and when using oral contraceptive pills. Erythema nodosum occurs among $4.6 \%$ of pregnant women [1].

The role of sex hormones in the aetiopathology of EN and its influence on the immunological system are not sufficiently known. Contraceptive pills were described as the most common drug causing EN, and reduced incidence of EN after the 1980s was observed, when low-oestrogen contraceptive drugs were introduced [4]. 
One mechanism of estrogens' influence on the immunological system is modulating it to increase the production of cytokines by T-cells and macrophages. In vitro research on mouse models showed that the supply of estrogens results in an increasing number of cells producing inflammatory cytokines such as interleukin 10 (IL-10) and IL-6. It confirmed the earlier research by Dayan et al. which revealed that the use of tamoxifen and anti-estrogens leads to elevated levels of IL-2 and IFN- $\gamma$ and to decreased levels of IL-10, IL-1 and TNF- $\alpha$ [18]

Some researchers argue that more important than the levels of estrogens and progesterone in the aetiology of EN are the proportions of these two hormones, as there has been no description of any cases of EN among women treated with high-dose estrogens therapy in breast cancer treatment. Erythema nodosum usually occurs in the first trimester of pregnancy when the level of progesterone is lower than in the next stage of gestation. Administration of tamoxifen and anti-estrogens such as aromatase inhibitors was associated with occurrence of the EN [4]. This raises the suspicion that it may have a correlation with the aforementioned elevated level of IFN- $\gamma$, caused by use of tamoxifen, which takes part in the inflammatory response with formation of granuloma, as it happens in erythema nodosum [19]. Interferon $\gamma$ plays a role in gestation as well, where it is produced by NK cells of the uterus, leading to dilatation and thickening of spiral arteries in order to provide the best nutrition to the fetus [20].

Occurrence of erythema nodosum in one of several gestations of the same woman or only once in many cycles of using contraceptive pills indicates that female sex hormones play mainly a role of modulators of the immune system, rather than directly impacting the pathomechanism of erythema nodosum.

\section{Enteropathies}

Skin disorders, after arthritis, uveitis and aphthous stomatitis, are one of the most common extra-intestina manifestations of inflammatory bowel diseases (IBD). Erythema nodosum is the most common cutaneous manifestation in patients with IBD, occurring in 4-15\% of Crohn's disease cases and in $3-10 \%$ of ulcerative colitis cases. It is also more prevalent in women.

It is presumed that skin lesions of erythema nodosum correlate with the activity of bowel disease, and in patients with Crohn's disease colonic involvement is observed more often [21].

Erythema nodosum usually parallels inflammatory bowel disease activity, although it may precede the diagnosis of IBD by up to five years. Treatment targeted at the underlying cause usually leads to resolution of the skin lesions [3].

There are some reports that erythema nodosum in patients with Crohn's disease is associated with the T-cell immune response to common antigens of intestinal and skin bacteria. In addition, there are suggestions that genetic factors also play a role in the pathogenesis of skin lesions in IBD - mainly variants of the TRAF3IP2 gene encoding a protein involved in inflammatory reactions by activating cytokines. The presence of ANCA and HLA-B27 antigen in patients with erythema nodosum and inflammatory bowel disease also argues in favour of the important role of genetic factors [22].

\section{Erythema nodosum as a paraneoplastic syndrome}

Erythema nodosum may be the first sign of an existent neoplastic disease. Paraneoplastic EN most often occurs with Hodgkin's lymphoma, non-Hodgkin's lymphoma and leukaemia, but it has also been linked to solid tumours $[12,23]$. The pathogenesis of malignancy-associated EN is unknown. It is suggested that paraneoplastic erythema nodosum is caused by an altered immune system response to a malignancy.

In cases associated with cancer, EN coincides or appears shortly before the diagnosis of the neoplasm. Skin lesions which recur chronically or persist for a long time require exclusion of an underlying malignant disease. Clinical features such as weight loss, fever, age at onset over 50, poor response to treatment and atypical laboratory investigations (e.g. hypergammaglobulinaemia, long-lasting anaemia, thrombocytopenia) are helpful in distinguishing paraneoplastic EN from non-neoplastic conditions. It is also known that erythema nodosum may indicate tumour relapse. For this reason, development of skin lesions in patients with previously treated malignancy requires oncological vigilance.

The early diagnosis of cancer in patients with paraneoplastic EN is very important and can be achieved by complete medical history taking, physical examination and age-appropriate cancer screening. Skin lesions usually respond to treatment of underlying cancer. Unfortunately, malignancy-associated erythema nodosum is considered to be a marker of poor prognosis [23-25].

\section{Summary}

It is important to remember that the diagnostic process in the case of erythema nodosum should be especially careful, because the cause of the skin lesion may be missed. In each case of erythema nodosum at least $X$-ray of the chest should be performed, and sometimes even computed tomography is necessary. Erythema no- 
dosum requires an interdisciplinary approach and exclusion of all underlying causes. EN can be treated by both dermatologists and rheumatologists - our clinical experience has shown that patients with erythema nodosum are referred more often to a rheumatologist than to a dermatologist if peripheral joints are involved.

The authors declare no conflict of interest.

\section{References}

1. Schwartz RA, Nervi SJ. Erythema nodosum: a sign of systemic disease. Am Fam Physician 2007; 75: 695-700.

2. Blake T, Manahan M, Rodins K. Erythema nodosum - a review of an uncommon panniculitis. Dermatol Online J 2014; 20: 22376.

3. Cowan JT, Graham MG. Evaluating the clinical significance of erythema nodosum. Patient Care 2005; 56-58.

4. Acosta KA, Haver MC, Kelly B. Etiology and therapeutic management of erythema nodosum during pregnancy: an update. Am J Clin Dermatol 2013; 14: 215-220.

5. Requena L, Sánchez YE. Erythema nodosum. Semin Cutan Med Surg 2007; 26: 114-122.

6. Sokolik R, Wiland P. Rumień guzowaty. In: Reumatologia. Wielka interna. Puszczewicz M (ed.). Medical Tribune Polska, Warszawa 2010; 226-230

7. Francès C, Boisnic S, Blétry O, et al. Cutaneous manifestations of Takayasu arteritis. A retrospective study of 80 cases. Dermatologica 1990; 181: 266-272.

8. Pascual-López M, Hernández-Núñez A, Aragüés-Montañés M, et al. Takayasu's disease with cutaneous involvement. Dermatology 2004; 208: 10-15.

9. Gupta M, Singh K., Lehl SS, Bhalla M. Recurrent erythema nodosum: a red flag sign of hidden systemic vasculitis. BMJ Case Rep 2013; 2013: 10.1136/bcr-2013-009507.

10. Misago N, Tada Y, Koarada S, Narisawa Y. Erythema nodosum-like lesions in Behçet's disease: a clinicopathological study of 26 cases. Acta Derm Venereol 2012; 92: 681-686.

11. Cebeci F, Onsun N, Ulusal HA, Inan B. The relationship between deep vein thrombosis and erythema nodosum in male patients with Behçet's disease. Eur Rev Med Pharmacol Sci.2014; 18: 3145-3148.

12. Kwiatkowska B, Przygodzka M, Filipowicz-Sosnowska A. Objawy reumatyczne w chorobach nowotworowych. J Oncol 2006; 56: 693-699.

13. Babamahmoodi F, Babamahmoodi A, Barani H, Delavarian L. Simultaneous occurrence of erythema nodosum in monozygotic twin sisters. Case Rep Med 2012; 2012: 109427.

14. Chen S, Chen J, Chen L, et al. Mycobacterium tuberculosis infection is associated with the development of erythema nodosum and nodular vasculitis. PLoS One 2013; 8(5): e62653.

15. Grzelewska-Rzymowska I. Sarkoidoza - choroba ogólnoustrojowa. Alergia 2012; 2: 23-31.

16. McDougal KE, Fallin MD, Moller D, et al.; and the ACCESS Research Group, Variation in the lymphotoxin- $\alpha /$ tumor necrosis factor locus modifies risk of erythema nodosum in sarcoidosis. J Invest Dermatol 2009; 129: 1921-1926.
17. Costabel U, Guzman J, Drent M. Diagnostic approach to sarcoidosis. Eur Respir Monograph 2005; 10: 259-260.

18. Verthelyir D. Sex hormones as immunomodulators in health and disease. International Immunopharmacology 2001; 6: 983-993.

19. Dayan M, Zinger H, Kalush F, et al. The beneficial effects of treatment with tamoxifen and anti-estradiol antibody on experimental systemic lupus erythematosus are associated with cytokine modulations. Immunology 1997; 90: 101-108.

20. Majkowska-Wojciechowska B. Zmiany układu immunologicznego wraz z wiekiem. Żywność dla Zdrowia 2010; 13: 10-13.

21. Huang BL, Chandra S, Shih DQ. Skin manifestations of inflammatory bowel disease. Front Physiol 2012; 3: 1-6.

22. Faulkes RE. Upper limb erythema nodosum: the first presentation of Crohn's disease. Clin Case Rep 2014; 2: 183-185.

23. Sendur OF. Paraneoplastic rheumatic disorders. Turk J Rheumatol 2012; 27: 18-23.

24. Racanelli V, Prete $M$, Minoia $C$, et al. Rheumatic disorders as paraneoplastic syndromes. Autoimmun Rev 2008; 7: 352-358.

25. Simon S, Azevedo SJ, Byrnes JJ. Erythema nodosum heralding recurrent Hodgkin 's Disease. Cancer 1985; 56: 1470-1472. 